\title{
Molienda asistida con microondas de un coque metalúrgico
}

\author{
Esteban Ruisánchez, Emilio J. Juárez-Pérez, Ana Arenillas, José M. Bermúdez, José Angel Menéndez ${ }^{\bowtie}$ \\ Grupo de Microondas y Carbones para Aplicaciones Tecnológicas. Instituto Nacional del \\ Carbón (INCAR). CSIC. Apartado 73, 33080 Oviedo, España \\ Autor para la correspondencia: angelmd@incar.csic.es
}

Enviado: 29 Enero 2014; Aceptado: 03 Febrero 2014

\begin{abstract}
RESUMEN: Los coques metalúrgicos están compuestos mayoritariamente de carbono grafítico (s2p2) y diferentes compuestos inorgánicos con distintas capacidades de absorber la radiación de microondas. Cuando se irradian con microondas fragmentos de estos coques, algunas partes de las partículas experimentan un rápido calentamiento, mientras otras no. Además, debido a que el coque presenta una cierta conductividad eléctrica, al ser irradiados con microondas se producen de forma puntual arcos eléctricos o microplasmas, dando lugar a puntos muy calientes. Como consecuencia de las diferentes dilataciones y tensiones producidas por el choque térmico, se producen en las partículas pequeñas grietas y microfisuras. Esto produce una mayor fragilidad en las partículas de coque y un incremento en la molturabilidad de las mismas. En el presente artículo se estudia la molienda de coque asistida con microondas y se evalúan las mejoras en la molturabilidad y el ahorro de energía producido.
\end{abstract}

PALABRAS CLAVE: Ahorro de energía; Choque térmico; Coque; Microondas; Molienda

Citation / Cómo citar este artículo: Ruisánchez, E., Juárez-Pérez, E.J., Arenillas, A., Bermúdez, J.M., Menéndez, J.A (2014) "Molienda asistida con microondas de un coque metalúrgico". Rev. Metal. 50(2): e013. doi: http://dx.doi. org/10.3989/revmetalm.013.

\begin{abstract}
Microwave-assisted grinding of metallurgical coke. Metallurgical cokes are composed of graphitic carbon (s2p2) and different inorganic compounds with very different capacities to absorb microwave radiation. Moreover, due to the electric conductivity shown by the metallurgical cokes, microwave radiation produces electric arcs or microplasmas, which gives rise to hot spots. Therefore, when these cokes are irradiated with microwaves some parts of the particle experiment a rapid heating, while some others do not heat at all. As a result of the different expansion and stress caused by thermal the shock, small cracks and micro-fissures are produced in the particle. The weakening of the coke particles, and therefore an improvement of its grindability, is produced. This paper studies the microwave-assisted grinding of metallurgical coke and evaluates the grinding improvement and energy saving.
\end{abstract}

KEYWORDS: Coke; Energy saving; Grindability; Microwave; Thermal shock

Copyright: $(\mathbb{C} 2014$ CSIC. This is an open-access article distributed under the terms of the Creative Commons AttributionNon Commercial (by-nc) Spain 3.0 License.

\section{INTRODUCCIÓN}

La operación de molienda en la industria pesada está asociada a un alto coste energético y de mantenimiento de equipos (Mular y Bhapp, 1982; Babich et al., 1996; Wills y Napier-Munn, 2006). En general, la operación de molienda de materiales duros, produce un importante desgaste mecánico en los molinos, por efecto del rozamiento y abrasión del material tratado, lo que supone unos costes de 
mantenimiento que en ocasiones pueden ser considerables. La minimización de estos costes es objeto de varios estudios que abarcan muchos aspectos del proceso (Babich et al., 1996; Chenje et al., 2003; Chenje et al., 2004).

Las industrias siderúrgica (Hearson, 1922) y del cemento (Stoltze, 2000) necesitan de la molienda de coque metalúrgico. Es necesario considerar cualquier técnica que prevea disminuir el gasto de la molienda de coque, que se realiza de forma intensiva en estas industrias.

Por un lado, la aplicación de un tratamiento térmico de forma convencional para asistir la molienda de minerales ya fue estudiada en el siglo pasado (Holman, 1926), dado que es conocido que al aplicar calor se promueve la fractura del mineral facilitándose con ello la molienda. Sin embargo, la mayoría de las veces el proceso es energéticamente desfavorable por lo que se desestima su uso (Fitzgibbon y Veasey, 1990). Por otro lado, el uso de las microondas, para el aporte de energía en procesos industriales de gran volumen de producción, como es el caso del presente trabajo, requiere ser estudiado experimentalmente (Krestou y Panias, 2004; Menéndez et al., 2010).

La principal ventaja del empleo de microondas frente al calentamiento por medios convencionales, es que el primero afecta específicamente a cada material, así, podemos encontrarnos con materiales que son transparentes a las microondas y que apenas aumentan la temperatura y otros que absorben la radiación y son rápidamente calentados (Church et al., 1988). Este hecho ha causado gran interés y en la literatura podemos encontrar muchos ejemplos que aprovechan esta ventaja de las microondas. Dos ejemplos que representan los extremos de la utilización de microondas en la molienda son, por un lado, la molienda de la cromita asistida por microondas, en la que el tratamiento mostró tener unos efectos limitados y una pobre variación del índice de trabajo (Güngör y Atalay, 1998); y por otro la molienda de la kimberlita para la extracción del diamante, en la que el proceso asistido por microondas presenta la ventaja de que sólo la ganga es calentada, de manera que el diamante es menos dañado que en una molienda convencional, al no verse afectado por la radiación y necesitar menos tiempo de residencia en el molino. Esto lleva a obtener piezas menos dañadas y por tanto de mayor valor (Didenko et al., 2005). En este último caso, el producto obtenido justifica el uso de las microondas para facilitar la molienda. Pero sin llegar a tales extremos, cuando la liberación de un mineral sea difícil económicamente, por métodos tradicionales, se puede intentar aplicar este pretratamiento no convencional (Kingman y Rowson, 1998). La efectividad y éxito de un pretratamiento con microondas depende específicamente del mineral o material a tratar, esto es, composición química (si hay o no un buen absorbente de microondas), tamaño de las partículas y forma de distribución de éstas en la matriz (Kingman et al., 2000; Kingman et al., 2004). Este es el caso de la molienda asistida con microondas de carbones, cuyos resultados han demostrado una reducción de hasta un $50 \%$ en el índice de trabajo en carbones de bajo rango debido al mayor contenido de humedad. Este contenido en agua es el principal absorbente de radiación en los carbones además de otros minerales como la pirita. La liberación de la primera y la expansión de los segundos provocará la fractura de la piezas de carbón que posteriormente facilitarán su molienda (Marland et al., 2000; Lester y Kingman, 2004). Recientemente, ya se ha informado sobre experimentos con la molienda de carbones en parámetros de operación similares a los que se usarían industrialmente (Lester et al., 2005).

El coque, mayoritariamente compuesto de carbono grafítico (s2p2), a diferencia del carbón que por sí sólo es un pobre absorbente (v.s.), tratado bajo microondas presenta el comportamiento denominado conductor. De hecho una exposición prolongada a esta radiación durante algunos segundos lleva a la formación de arcos eléctricos o microplasmas (Menéndez et al., 2010).

El objetivo de este estudio es por tanto investigar el efecto de la irradiación de microondas sobre un coque y examinar las ventajas que esto conlleva en una posterior molienda. Dadas las limitaciones de los equipos de laboratorio, en comparación con la molienda industrial, los resultados obtenidos han de tomarse como meramente cualitativos, aunque de una importancia crucial para discernir si llevar a cabo o no el escalado a la industria y sobre todo, el principal objetivo: hallar experimentalmente una primera aproximación de la mínima cantidad de energía suministrada a un coque vía microondas para una eficiencia máxima en la molienda.

\section{MATERIALES Y MÉTODOS}

\subsection{Material de partida}

Un coque procedente de la empresa Arcelor Mittal (Asturias) fue uniformado granulométricamente para elaborar unas muestras de ensayo que contuvieran un $25 \%$ en peso de cada una de las siguientes cuatro fracciones de tamaño de partículas: 1-2, 2-3, 3-4 y 4-5 mm (Lester et al., 2005). De esta forma todas las muestras usadas pueden considerarse uniformes en lo que respecta a la granulometría de partida. El indicador escogido para evaluar el grado de molienda es el porcentaje de finos obtenido tras la molienda (partículas de tamaño inferior a $1 \mathrm{~mm}$ ).

\subsection{Molienda}

La molienda en el laboratorio de las distintas muestras de coque se realiza en un molino de bolas, modelo Retsch MM400. El molino posee dos cilindros de acero de $3,5 \mathrm{~cm}$ de diámetro y $6,5 \mathrm{~cm}$ de 


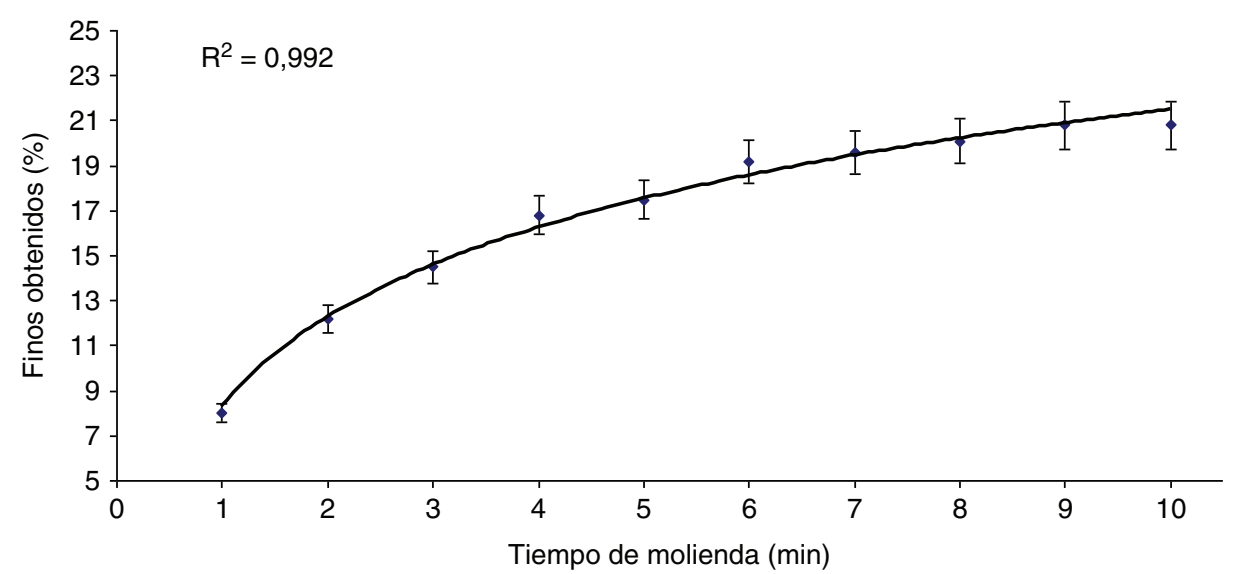

FIgURA 1. Molienda a $10 \mathrm{~Hz}$. Relación entre el porcentaje de finos y el tiempo de molienda, con barras de error.

largo, con una bola de acero de $1 \mathrm{~cm}$ de diámetro en su interior y que se mueve libremente a una frecuencia controlada por el usuario. Con el fin de minimizar el gasto energético se estudiaron tres frecuencias de trabajo: 5, 10 y $20 \mathrm{~Hz}$, durante diferentes tiempos totales de molienda y con distintas cargas del coque en los cilindros. De estos experimentos previos se establece que la frecuencia óptima es $10 \mathrm{~Hz}$ debido a que la cantidad de finos obtenida con una frecuencia de $5 \mathrm{~Hz}$ es muy escasa y la frecuencia de $20 \mathrm{~Hz}$ no difiere sustancialmente respecto a la operación a $10 \mathrm{~Hz}$. Para utilizar una frecuencia de $10 \mathrm{~Hz}$ el molino necesita una potencia de $63 \mathrm{~W}$.

Cada cilindro se carga con $5 \mathrm{~g}$ de coque. Así, cada resultado experimental corresponde a la media aritmética del resultado obtenido en cada uno de los cilindros. La relación existente entre el porcentaje de finos y el tiempo de molienda se ajusta a una curva logarítmica, como se aprecia en la Figura 1.

Para comparar los diferentes pretratamientos con microondas se ha escogido un tiempo de 3 minutos. En este tiempo, el coque molido sin pretratamiento presenta un 13,2 $\pm 0,7 \%$ en peso de finos. Para este porcentaje de finos se puede evaluar la molienda de forma óptima, ya que a tiempos de molienda más cortos el porcentaje de finos será menor y más aleatorio, y a tiempos mayores, un porcentaje de finos demasiado grande enmascara el beneficio del pretratamiento con microondas, pues para un tiempo infinito de molienda, los dos procesos darán el mismo porcentaje de finos.

\subsection{Pretratamiento con microondas}

El microondas utilizado para el pretratamiento de las muestras de coque es de tipo doméstico, modificado para trabajar a una potencia constante de $700 \mathrm{~W}$, lo que representa $1050 \mathrm{~W}$ de consumo eléctrico de la red.

El pretratamiento del coque en el microondas se realiza en la mayoría de los casos sobre $20 \mathrm{~g}$ de muestra, distribuidos en una superficie circular de $113 \mathrm{~cm}^{2}$ sobre un aislante de lana de cuarzo situado en la parte central de la cavidad del microondas. El espesor de la capa de coque es de unos $10 \mathrm{~mm}$. Se utilizan $20 \mathrm{~g}$ de muestra para facilitar el número elevado de ensayos realizados. Se hicieron pruebas para buscar el límite de masa para que el pretratamiento sea efectivo, cargando el microondas hasta con $500 \mathrm{~g}$ de muestra, como se muestra más adelante. En todos los casos la muestra se situó centrada sobre la base de la cuba microondas con una disposición circular. Con una muestra de $500 \mathrm{~g}$, la superficie ocupada es de $254 \mathrm{~cm}^{2}$ con un espesor de la capa de coque de $80 \mathrm{~mm}$. La capacidad del microondas no permitió usar cantidades superiores a $500 \mathrm{~g}$.

Se ha observado que el tiempo total de irradiación al que se someten las muestras en el microondas y el modo en que se aplicó durante este tiempo son factores determinantes en la cantidad de finos obtenidos en la molienda posterior. Los tiempos totales de irradiación fueron de: 2 , $3,6,9,18$ y 30 segundos. En cuando al modo de aplicación de la irradiación se han estudiado los siguientes ciclos:

- $\quad 1 \mathrm{~s} \mathrm{ON}-3 \mathrm{~s}$ OFF: la muestra se somete a una irradiación de 1 segundo seguida de un periodo de reposo de 3 segundos. El ciclo se aplica sucesivamente hasta completar el tiempo de irradiación total programado.

- $\quad 3$ s ON -3 s OFF: este ciclo se basa en la irradiación de la muestra durante 3 segundos seguida de un periodo de reposo de 3 segundos.

- 9 s ON -3 s OFF: periodo de irradiación de 9 segundos con un reposo posterior de 3 segundos. La aplicación de este ciclo supone un cierto riesgo de incendio de la muestra, por lo que esta modalidad no es la recomendable. No se han estudiado ciclos con un periodo de irradiación superior debido a este riesgo. 
Para una misma cantidad de energía microondas aplicada, si se utilizan distintos tiempos de reposo entre 1 y 7 segundos, los resultados son similares, en lo que respecta a la mejora de la molienda. Sin embargo, el no dejar ningún tiempo de reposo, da lugar a peores resultados además del riesgo de incendio que ello conlleva. Esto se debe probablemente a un menor efecto del shock térmico al que dan lugar las reiteradas secuencias de encendido y apagado de las microondas. El shock térmico producido por estas secuencias de encendido y apagado provocará en el coque la aparición de pequeñas grietas y microfisuras tanto interna como superficialmente, lo que provocará un debilitamiento en su estructura y por tanto menos resistencia a la abrasión.

\section{RESULTADOS Y DISCUSIÓN}

\subsection{Limitaciones del horno microondas}

El horno microondas utilizado presenta dos limitaciones. Por una parte la eficiencia de estos equipos, según la bibliografía, está entre el 10 y el $40 \%$ (Schubert et al., 2009), pero en las experiencias que se han llevado a cabo, a nivel de laboratorio, con distintas cantidades de agua, se obtuvo una eficiencia del $56 \%$. Estas pruebas se realizaron ocupando con agua una superficie y distribución similar a la del coque, irradiando con microondas y midiendo, con un termopar, el aumento de temperatura con respecto al tiempo. Este dato, junto al calor específico del agua, permite calcular la energía que ha sido necesaria utilizar para elevar la temperatura. Conociendo la potencia suministrada por el microondas, se puede hallar la eficiencia del equipo. Por otra parte, en el horno utilizado no fue posible emplear tiempos de irradiación inferiores a 1 segundo, tal como realizan diversos autores para otros materiales. Dichos autores emplean tiempos de calentamiento inferiores a 1 segundo pero suministran cantidades de energía muy altas (Lester y Kingman, 2004; Lester et al., 2005; Lester et al., 2006), difiriendo de los experimentos realizados en este estudio donde se suministró menos energía durante periodos más largos de tiempo.

\subsection{Mejora del porcentaje de finos con la molienda}

Para observar la mejora producida en la molienda, en la Figura 2 se muestra el incremento del porcentaje de finos obtenidos en diferentes alícuotas $(20 \mathrm{~g})$ de coque irradiadas con microondas respecto a los obtenidos en una muestra sin pretratar, según el modo y tiempo de irradiación.

Tal y como se observa en la Figura 2, existe una mejora en la operación en todos los casos, independientemente de la modalidad y el tiempo total irradiado.

El tratamiento que mejores resultados proporciona es el de 18 segundos de irradiación en la modalidad 9 s ON -3 s OFF con un incremento en la producción de finos del $28 \%$. Sin embargo se ha descartado este modo de operación, ya que además de resultar energéticamente menos conveniente, conlleva ciertos riesgos de incendio de la muestra. Por lo tanto, la opción más atractiva sería la modalidad de operación de $1 \mathrm{~s}$ ON -3 s OFF, la cual da lugar a un incremento en los finos obtenidos tras la molienda entre el 15-25\%. Esta modalidad resulta más eficaz para todos los tiempos de irradiación totales que la modalidad $3 \mathrm{~s} \mathrm{ON}-3 \mathrm{~s}$ OFF. Esto concuerda con la hipótesis apuntada anteriormente del efecto del shock térmico, ya que se produce un número mayor de secuencias de encendido-apagado. Así, una vez que se produce el incremento brusco de temperatura en el interior de las partículas, un aumento en el tiempo de radiación consigue simplemente mantener una temperatura elevada en el seno de la muestra que está

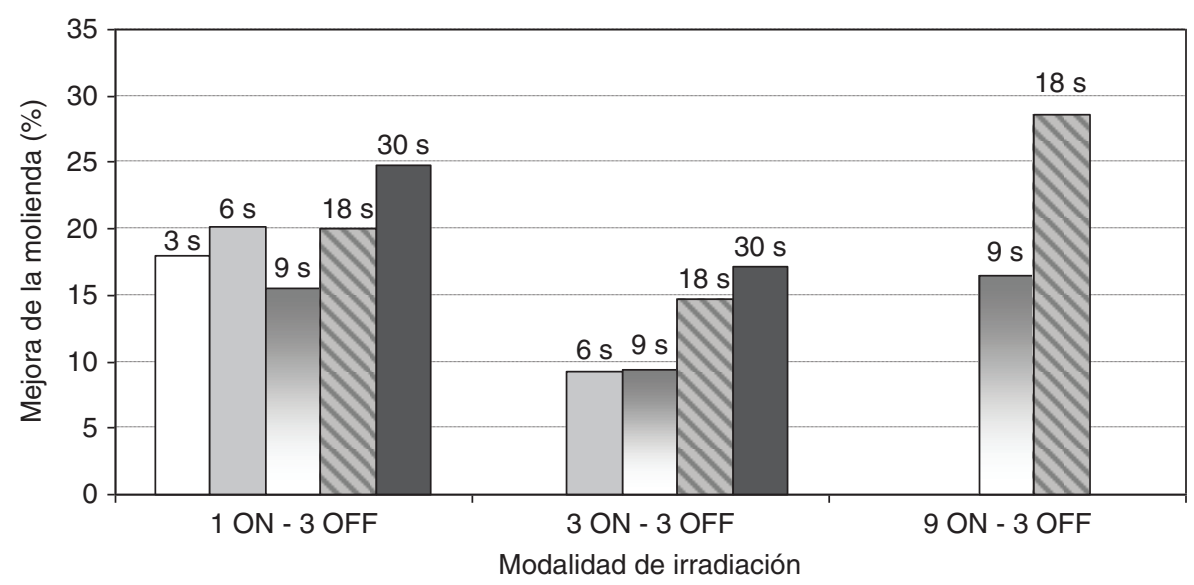

FIGURA 2. Mejora de la molienda, expresada como incremento en el porcentaje de finos obtenidos con respecto al blanco, para diferentes modalidades de irradiación con microondas. 

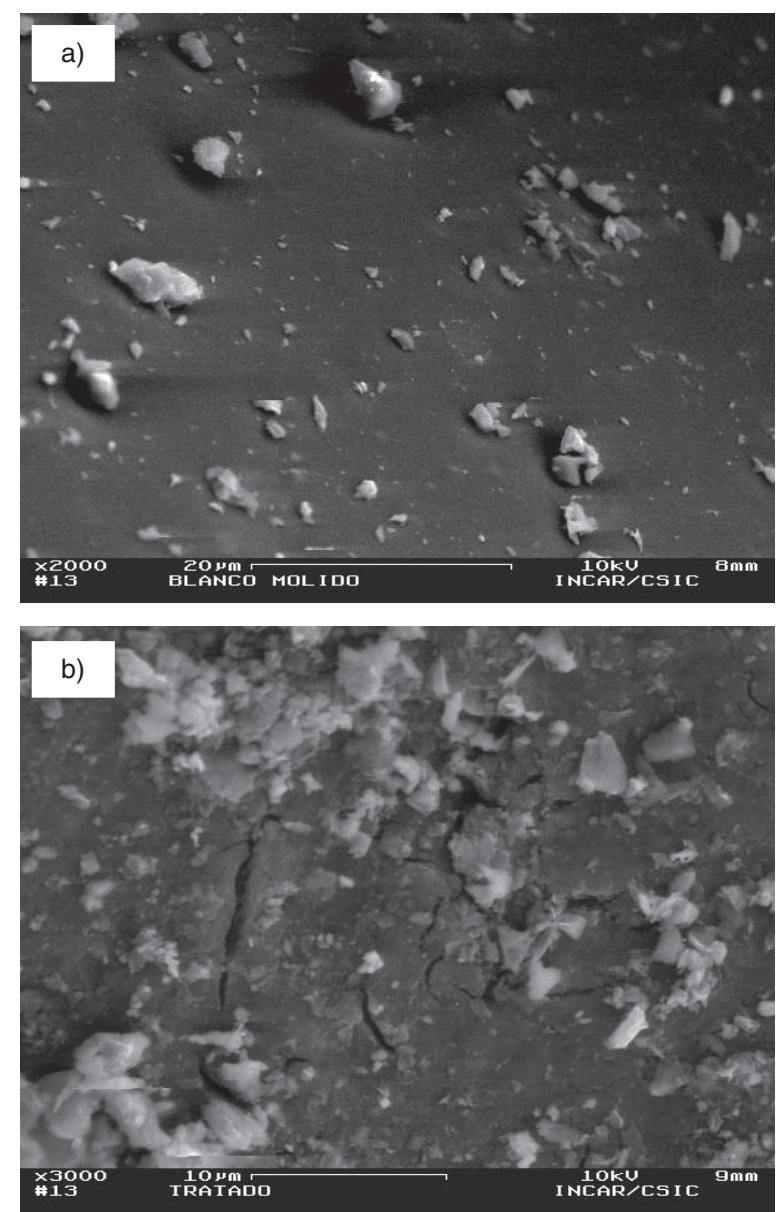

FigurA 3. Microfotografías de: (a) una partícula de coque sin tratar y (b) una partícula de coque sometido al tratamiento en microondas durante 3 segundos en la modalidad $1 \mathrm{~s} \mathrm{ON}-3 \mathrm{~s} \mathrm{OFF}$

siendo tratada. Esto resulta menos eficaz, a la hora de producir el debilitamiento de las partículas, que el shock térmico por aumentos y descensos bruscos de temperatura que producen los ciclos rápidos de encendido y apagado de las microondas.

Una prueba de que el efecto de la irradiación con microondas da lugar a una microfisuración de las partículas de coque puede observarse en la Figura 3, en la que se muestran microfotografías obtenidas con un microscopio electrónico de barrido (MEB) de la microestructura de una partícula de coque sin tratar y otra después de haber sido sometida al tratamiento en microondas durante 3 segundos en la modalidad 1s ON -3 s OFF. Se han visualizado los materiales sin necesidad de pretratamiento previo en un SEM DMS-942, (Zeiss, Germany) equipado con un detector EDX (Link-Isis II, Oxford, UK).

La partícula sometida al tratamiento en microondas presenta un agrietamiento mucho mayor que en el caso de la partícula no sometida a dicho tratamiento. Si bien aquí se muestra un único ejemplo, el estudio llevado a cabo en el MEB reveló que la incidencia de microfisuras, en el seno de las partículas, es notablemente superior en el caso de las muestras tratadas en el microondas, cualquiera que fuese el modo de tratamiento.

\subsection{Mejora en el tiempo de molienda}

El incremento del porcentaje de finos obtenidos citado anteriormente, representa una mejora en la molienda, lo cual, a su vez, supone un ahorro de tiempo. Es decir, para obtener la misma cantidad de finos que se obtienen después de un pretratamiento con microondas, una muestra, no sometida a este pretratamiento, requiere de un tiempo de molienda más prolongado. Dicho tiempo puede determinarse por interpolación en la curva de la Figura 1.

En la Figura 4 se muestra este ahorro de tiempo en la molienda (expresado en \%) para los diferentes tiempos de irradiación en la modalidad 1s $\mathrm{ON}$ -3 s OFF. Como se puede apreciar, a excepción del experimento con tiempo de irradiación de 9 segundos, que es considerado anómalo, un mayor tiempo de exposición a las microondas da lugar a una mayor eficacia de la molienda, o si se prefiere a un mayor ahorro de tiempo. Sin embargo, mientras que 2 y 3 segundos de exposición dan lugar a incrementos en el ahorro de tiempo de un $20 \%$ y $40 \%$ respectivamente, un aumento en el tiempo de exposición da lugar a incrementos mucho menores en el ahorro del tiempo.

Estos resultados son comparables a los obtenidos para otros materiales (Marland et al., 2000; Kingman et al., 2004; Lester y Kingman, 2004). Esto vendría a suponer, que una vez conseguido el efecto inicial de fisuración de la partícula por shock térmico, cada vez resulta más difícil incrementar el deterioro de la partícula usando este procedimiento. A la vista de estos resultados, 3 segundos de irradiación parece ser la forma más conveniente de operación, al menos en las condiciones experimentales usadas en este trabajo. A escala industrial, el ahorro en tiempo de molienda supondrá, además de un menor consumo de energía en la molienda, un ahorro en los gastos de mantenimiento de los molinos (Mular y Bhapp, 1982; Wills y Napier-Munn, 2006).

\subsection{Límite de la cantidad de coque tratada}

Asimismo se ha estudiado la cantidad de coque que se puede tratar en el microondas obteniendo un porcentaje de finos similar. En la Figura 5 se muestran los resultados. Tal y como se puede apreciar, un aumento de la cantidad de coque, hasta alcanzar la capacidad máxima de tratamiento i.e., 500 g, no produce un cambio sustancial en el porcentaje de finos obtenidos en la molienda. La línea horizontal marca el porcentaje de finos obtenido en una muestra que no ha recibido tratamiento previo a su molienda. 


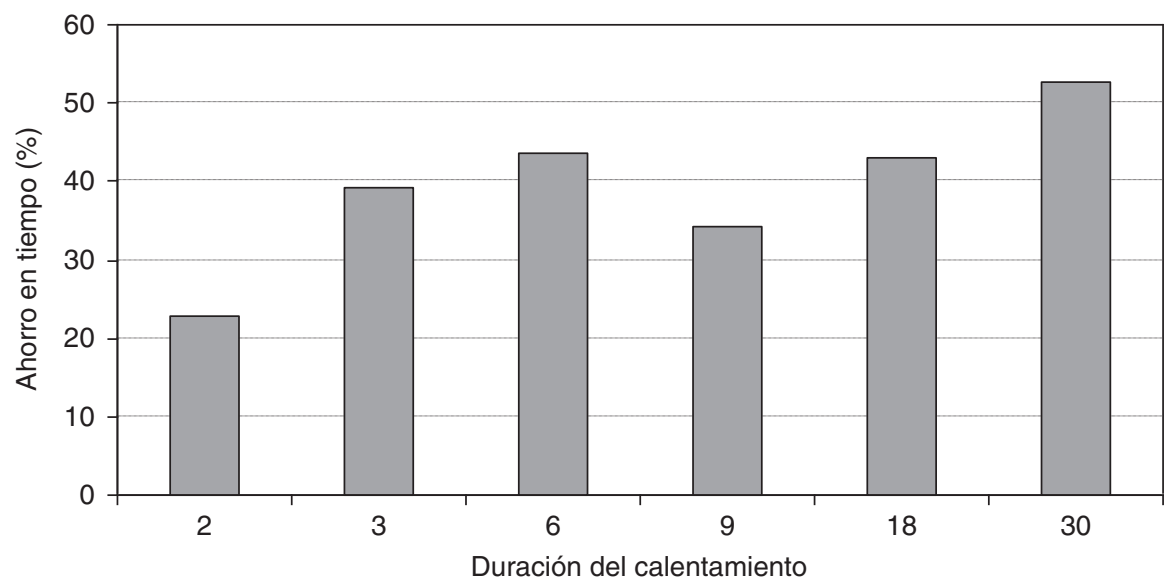

FIgURA 4. Ahorro de tiempo en la molienda para los diferentes tiempos de irradiación totales usando ciclos $1 \mathrm{~s}$ ON $-3 \mathrm{~s}$ OFF.

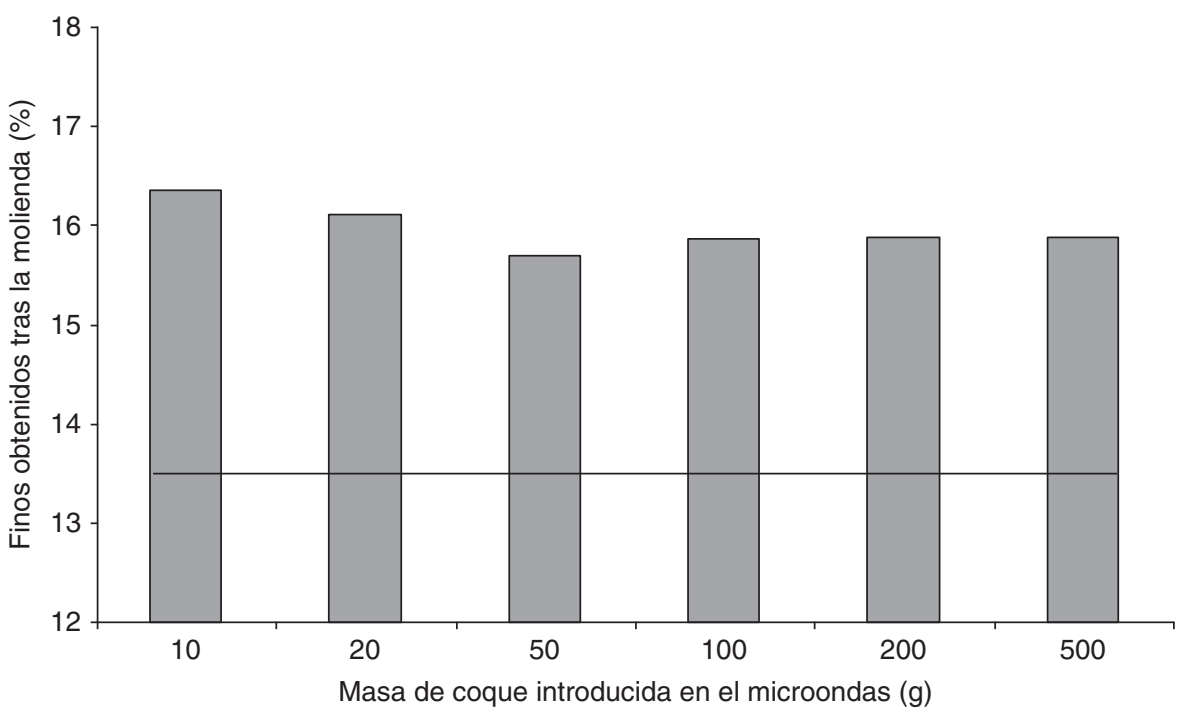

FIGURA 5. Variación del porcentaje de finos producidos con la masa de coque tratada utilizando el modo de funcionamiento $1 \mathrm{~s} \mathrm{ON}-3 \mathrm{~s}$ OFF durante 3 segundos.

\subsection{Balance energético}

Para poder expresar el ahorro en tiempo en ahorro de energía, se utilizó un medidor de potencia para calcular la energía que consume por segundo y por gramo, tanto en el microondas $\left(1,4 \mathrm{~J} \mathrm{~s}^{-1} \mathrm{~g}^{-1}\right)$ como en el molino $\left(6,3 \mathrm{~J} \mathrm{~s}^{-1} \mathrm{~g}^{-1}\right)$. Con el tiempo que se ha ahorrado de molienda tras el pretratamiento con microondas podemos estimar el ahorro energético en el molino. A esta cantidad habría que descontar la energía que ha sido consumida por el microondas en la fase de pretratamiento, obteniendo así un balance neto de ahorro o gasto de energía de todo el proceso. Los resultados se expresan en la Figura 6.

Se puede apreciar cómo el ahorro energético del $43 \%$ se alcanza con un máximo local a los 6 segundos. Cuanto más tiempo dure el tratamiento, mayor mejora de molienda se producirá, pero a partir de 3 segundos, un incremento en el tiempo de exposición da lugar a incrementos mucho menores en el ahorro del tiempo de molienda.

Los tratamientos de pocos segundos de radiación con microondas llevan asociados bajos consumos energéticos y un ahorro de tiempo en el uso del molino, por lo que el proceso global resulta energéticamente favorable. A partir de los 6 segundos, que marcan un punto de inflexión tal como se muestra en la Figura 6, el ahorro de tiempo no aumenta sustancialmente, mientras que el consumo producido por el microondas es cada vez mayor, por lo que el balance energético neto del proceso es menos positivo. Con tratamientos durante periodos de tiempo 


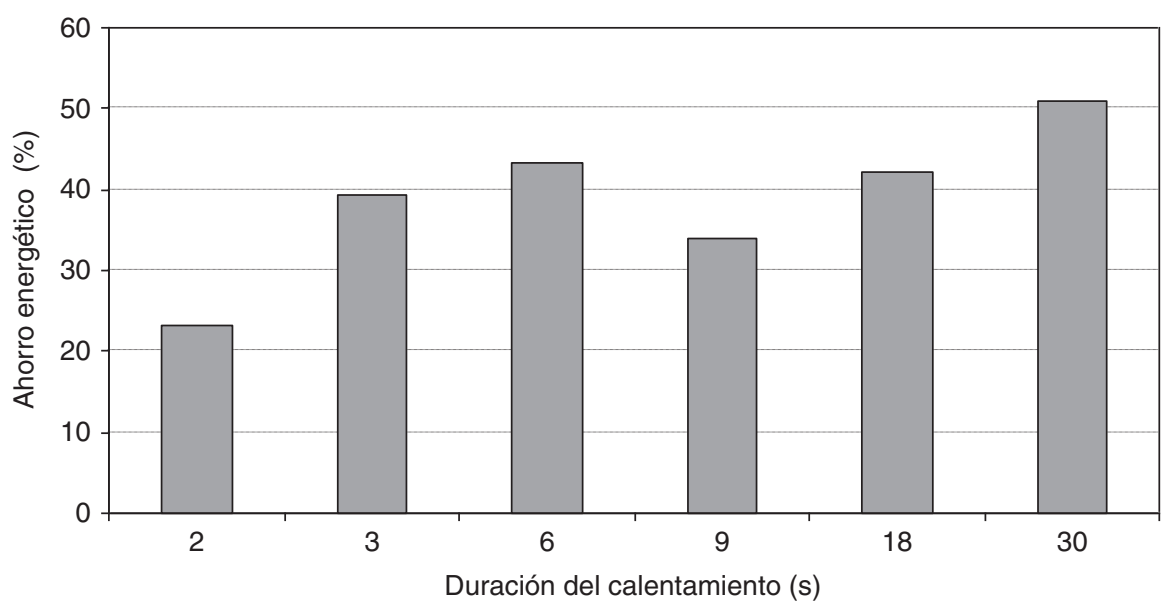

Figura 6. Variación del ahorro energético en la molienda con el tiempo total de irradiación en el modo 1s ON - 3 s OFF.

de irradiación más largos no se produce una mejora en la molienda; a partir de ese momento el balance energético empezaría a decrecer llegando a ser negativo a un tiempo determinado.

\section{CONCLUSIONES}

La estructura del coque tratado en microondas es debilitada por la aparición de microfracturas internas. Esto conduce a una mejora en la molienda que puede observarse por una mayor cantidad de finos (partículas de tamaño inferior a $1 \mathrm{~mm}$ ) producidos. Por tanto, lograr el mismo grado de molienda conlleva menor tiempo de uso del molino, lo que supondrá un menor gasto de energía y principalmente un menor gasto en mantenimiento de los molinos. Como contrapunto, existe un gasto energético debido al uso de los microondas.

Como conclusión se puede indicar que el mejor modo de funcionamiento sería el 1s ON - 3 s OFF durante 3 segundos (3 ciclos), llegando a obtenerse un ahorro en tiempo y de energía neto del 39\% en ambos casos.

\section{AGRADECIMIENTOS}

Los autores agradecen a Arcelor Mittal (Asturias) la financiación del trabajo.

\section{REFERENCIAS}

Babich, A., Yaroshevskii, S., García, L., Formoso, A., Cores, A., Isidro, A., Ferreira, S. (1996). Technological improvements in the pulverized coal injection process in the blast furnace. Rev. Metal. 32 (2), 103-116. http://dx.doi.org/10.3989/ revmetalm.1996.v32.i2.921.

Chenje, T.W., Simbi, D.J., Navara, E. (2003). Wear performance and cost effectiveness - A criterion for the selection of grinding media for wet milling in mineral processing operations. Miner. Eng. 16 (12), 1387-1390. http://dx.doi.org/ 10.1016/j.mineng.2003.08.009.
Chenje, T.W., Simbi, D.J., Navara, E. (2004). Relationship between microstructure, hardness, impact toughness and wear performance of selected grinding media for mineral ore milling operations. Mater. Des. 25 (1), 11-18. http:// dx.doi.org/10.1016/S0261-3069(03)00168-7.

Church, R.H., Webb, W.E., Salsman, J.B. (1988). Dielectric properties of low-loss minerals. U. S. Bureau of Mines. Report of Investigations. Report 9194.

Didenko, A.N., Zverev, B.V., Prokopenko, A.V. (2005). Microwave fracturing and grinding of solid rocks by example of kimberlite. Doklady Physics 50 (7), 349-350. http://dx.doi.org/ $10.1134 / 1.2005358$.

Fitzgibbon, K.E., Veasey, T.J.(1990). Thermally assisted liberation - a review. Miner. Eng. 3 (1-2), 181-185. http://dx.doi.org/ 10.1016/0892-6875(90)90090-X.

Güngör, A., Atalay, Ü. (1998). Microwave processing and grindability. Innovations in Mineral and Coal Processing. Innovations in Mineral and Coal Processing: Proceedings of the 7th International Mineral Processing Symposium, Istanbul, 13-16.

Hearson, H.R. (1922). The Manufacture of Iron and Steel; E \& F. N. Spon Ltd., London, UK.

Holman, B.W. (1926). Heat treatment as an agent in rock breaking. Trans. Inst. Min. Metall. 36, 219-234.

Kingman, S.W., Rowson, N.A. (1998). Microwave treatment of minerals - a review. Miner. Eng. 11 (11), 1081-1087. http:// dx.doi.org/10.1016/S0892-6875(98)00094-6.

Kingman, S.W., Vorster, W., Rowson, N.A. (2000). The influence of mineralogy on microwave assisted grinding. Miner. Eng. 13 (3), 313-327. http://dx.doi.org/10.1016/S0892-6875 (00)00010-8.

Kingman, S.W., Jackson, K., Cumbane, A., Bradshaw, S.M., Rowson, N.A., Greenwood, R. (2004). Recent developments in microwave assisted comminution. Int. J. Miner. Process. 74 (1-4), 71-83. http://dx.doi.org/10.1016/j.minpro. 2003.09.006.

Krestou, A., Panias, D. (2004). 1st International Conference on Advances in Mineral Resources Management and Environmental Geotechnology Hania, Greece, 215-220.

Lester, E., Kingman, S. (2004). The effect of microwave preheating on five different coals. Fuel 83 (14-15), 1941-1947. http://dx.doi.org/10.1016/j.fuel.2004.05.006.

Lester, E., Kingman, S., Dodds, C. (2005). Increased coal grindability as a result of microwave pretreatment at economic energy inputs. Fuel 84 (4), 423-427. http://dx.doi.org/ 10.1016/j.fuel.2004.09.019.

Lester, E., Kingman, S., Dodds, C., Patrick, J. (2006). The potential for rapid coke making using microwave energy. Fuel 85 (14-15), 2057-2063. http://dx.doi.org/10.1016/j. fuel.2006.04.012. 
Marland, S., Han, B., Merchant, A., Rowson, N. (2000). The effect of microwave radiation on coal grindability. Fuel 79 (11), 1283-1288. http://dx.doi.org/10.1016/S0016-2361 (99)00285-9.

Menéndez, J.A., Arenillas, A., Fidalgo, B., Fernández, Y., Zubizarreta, L., Calvo, E.G., Bermúdez, J.M. (2010). Microwave heating processes involving carbon materials. Fuel Process. Technol. 91 (1), 1-8. http://dx.doi.org/10.1016/j. fuproc.2009.08.021

Mular, A.L., Bhappu, R.B. (1982). Diseño de plantas de proceso de minerales, Madrid.
Schubert, U.S., Hoogenboom, R., Wilms, T.F.A., Erdmenger, T. (2009). Microwave-assisted chemistry: a closer look at heating efficiency. Aust. J. Chem. 62 (3), 236-243. http://dx.doi. org/10.1071/CH08503.

Stoltze, S. (2000). The use of pet coke in cement manufacturing plants: Presentation of industrial cases of grinding and firing of pet coke. 11th International Cement Conference Hammamet, Tunisie, 9 .

Wills, B.A., Napier-Munn, T. (2006). Wills mineral processing technology: an introduction to the practical aspects of ore treatment and mineral recovery. Butterworth-Heinemann, 2006. 